\title{
FINITENESS OF MORDELL-WEIL GROUPS OF GENERIC ABELIAN VARIETIES
}

\author{
BY ALICE SILVERBERG
}

In a series of papers in the 1960s Shimura studied analytic families of abelian varieties with fixed polarization, endomorphism, and level structure. The isomorphism classes of abelian varieties in such a family are in one-to-one correspondence with the points of $D / \Gamma$, where $D$ is a symmetric domain and $\Gamma$ is a discontinuous group of transformations of $D$. Shimura constructed a fibre system $(V, W)$ where the base $V$ is analytically isomorphic to $D / \Gamma$, the fibres are the abelian varieties in the family, and $V$ and $W$ are quasi-projective varieties. The fibre $A$ over the generic point of $V$ is an abelian variety defined over the function field $K$ of $V$. The main result of this announcement is that, under certain conditions on the endomorphism algebra structure, the group of points of $A$ defined over $K$ is finite. Using completely different techniques, Shioda [8] proved this result in the case in which $D$ is the complex upper half-plane and $\Gamma$ is a congruence subgroup of $\mathrm{SL}_{2}(Z)$.

The results in this note are an extension of part of the author's Ph.D. thesis [9]. Details will appear elsewhere. I would like to express my sincere thanks to my thesis advisor, Professor Goro Shimura.

1. Let $F$ be an arbitrary totally real number field of degree $g$ over the rational number field $\mathrm{Q}$. Let $L$ be either (a) the field $F$, (b) a totally indefinite quaternion algebra over $F$ (and view $L$ as embedded in $M_{2}(\mathbb{R})^{g}$ ), or (c) a totally imaginary quadratic extension $K$ of $F$. Let $\Phi$ be a representation of $L$ by complex matrices of degree $n$ so that $\Phi+\bar{\Phi}$ is equivalent to a rational representation of $L$, and $\Phi(1)=1_{n}$ (writing $1_{n}$ for the identity matrix of size $n)$. Assume that $[L: \mathbb{Q}]$ divides $2 n$, and let $m=2 n /[L: \mathbb{Q}]$. In (c), if $\tau_{1}, \ldots, \tau_{g}, \bar{\tau}_{1}, \ldots, \bar{\tau}_{g}$ are the distinct embeddings of $K$ in the complex number field $\mathbb{C}$, write $r_{\nu}$ and $s_{\nu}$, respectively, for the multiplicities of $\tau_{\nu}$ and $\bar{\tau}_{\nu}$ in $\Phi$ (then $r_{\nu}+s_{\nu}=m$ ). Suppose $T \in M_{m}(L)$ satisfies ${ }^{\mathrm{t}} T^{\rho}=-T$, where ${ }^{\mathrm{t}}$ is transpose on $M_{m}(L)$, and $\rho$ is complex conjugation on $K$ and transpose on each factor of $M_{2}(\mathbb{R})^{g}$. In (c), suppose $i T^{\tau_{\nu}}$ has the same signature as

$$
\left(\begin{array}{cc}
1_{r_{\nu}} & 0 \\
0 & -1_{s_{\nu}}
\end{array}\right)
$$

for every $\nu$. Let $\mathcal{M}$ be a lattice in $L^{m}$, and let $v_{1}, \ldots, v_{s}$ be elements of $L^{m}$. Let $\Omega$ denote the collection of data $\left(L, \Phi, \rho, T, \mathcal{M}, v_{1}, \ldots, v_{s}\right)$.

Suppose $A$ is an abelian variety with a polarization $C, \theta$ is an embedding of $L$ into $\operatorname{End}(A) \otimes Q$, and $t_{1}, \ldots, t_{s}$ are elements of $A$ of finite order.

Received by the editors July 31, 1984 .

1980 Mathematics Subject Classification. Primary 14K10, 14K22; Secondary 10D20.

(C) 1985 American Mathematical Society $0273-0979 / 85 \$ 100+\$ .25$ per page 
DEFINITION. $\left(A, C, \theta, t_{1}, \ldots, t_{s}\right)$ is a polarized abelian variety of type $\Omega$ if (1) there is a holomorphic mapping $\xi$ of $\mathbb{C}^{n}$ onto $A$ inducing an isomorphism of a complex torus $\mathbb{C}^{n} / Y$ onto $A$ satisfying $\xi(\Phi(a) u)=\theta(a) \xi(u)$ for every $u \in \mathbb{C}^{n}$ and $a \in \theta^{-1}(\operatorname{End}(A)) ;(2)$ if $\gamma$ is the involution of $\operatorname{End}(A) \otimes \mathbb{Q}$ determined by $C$, then $\theta(a)^{\gamma}=\theta\left(a^{\rho}\right)$ for every $a \in L ;(3)$ there is an $\mathbb{R}$-linear isomorphism $\eta$ of $\left(L \otimes_{\mathbb{Q}} \mathbb{R}\right)^{m}$ onto $\mathbb{C}^{n}$ such that $\eta(\mathcal{M})=Y, t_{\imath}=\xi\left(\eta\left(v_{\imath}\right)\right)$ for $i=1, \ldots, s$, and $\eta(a x)=\Phi(a) \eta(x)$ for every $a \in L$ and $x \in\left(L \otimes_{\mathbb{Q}} \mathbb{R}\right)^{m}$; and (4) $C$ determines a Riemann form $R$ on $\mathbb{C}^{n} / Y$ such that $R(\eta(x), \eta(y))=$ $\operatorname{tr}\left(x T^{\mathrm{t}} y^{\rho}\right)$ for every $x$ and $y$ in $\left(L \otimes_{\mathbb{Q}} \mathbb{R}\right)^{m}$.

Write $H_{r}$ for $\left\{\left.Z \in M_{r}(\mathbb{C})\right|^{\mathrm{t}} Z=Z ; \operatorname{Im}(Z)\right.$ is positive symmetric $\}$ and $H_{r, s}$ for $\left\{Z \mid\right.$ complex matrix with $r$ rows and $s$ columns; $1-Z^{\mathrm{t}} \bar{Z}$ is positive hermitian\}. Let $D$ be $H_{m / 2}^{g}$ in (a), $H_{m}^{g}$ in (b), and $H_{r_{1}, s_{1}} \times \cdots \times H_{r_{g}, s_{g}}$ in (c). The isomorphism classes of polarized abelian varieties of type $\Omega$ are in oneto-one correspondence with the points of $D / \Gamma$, where $\Gamma$ is a suitably defined group of transformations on $D$ (see $[\mathbf{3}]$ and $[\mathbf{4}]$ ). In [5] Shimura showed that for each $\Omega$, one can construct a fibre system $\mathcal{F}$ in which the base $V$ is analytically isomorphic to $D / \Gamma$ and the fibres are the polarized abelian varieties of type $\Omega$.

THEOREM 1. If $\operatorname{dim}(V) \geq 1$ then the group of points of the generic fibre defined over the function field of $V$ is finite.

The remainder of this paper is a sketch of the proof of Theorem 1.

2. The Mordell-Weil group of Theorem 1 is isomorphic to the group of rationally defined algebraic sections from the base $V$ to the fibre variety $W$. If $V$ is one-dimensional, one sees easily that these sections extend to global holomorphic sections. For higher dimensions we have the following result, which is a consequence of a result of Igusa (Theorem 6 of $[\mathbf{1}]$ ) when the base variety $V$ is compact.

PROPOSITION. Let $f$ be a rational section from $V$ to $W$. Then $f$ is defined at every point of $V$ so that $f$ gives a holomorphic section from $V$ to $W$.

When $\operatorname{dim}(V)=1$ and $V$ is compact, the second derivative of a holomorphic section is an automorphic form of weight three with respect to $\Gamma$. The Eichler-Shimura cohomology isomorphism (Theorem 8.4 of $[\mathbf{7}]$ ) can be used to show these automorphic forms are zero, and this then restricts the number of holomorphic sections. When $D$ is $H_{1}^{r}$ or $H_{1,1}^{r}$ with $r>1$, the use of the Eichler-Shimura cohomology isomorphism is replaced by the application of a theorem of Matsushima and Shimura (Theorem 3.1 of [2]), which says there are no automorphic forms of mixed weight with at least one nonpositive weight.

3. The cases of Theorem 1 discussed in $\S 2$ can be used to prove the theorem in the remaining cases. We select a large collection of embeddings of base varieties $V^{\prime}$, for which the theorem is known, into a variety $V$ for which we want to prove the theorem. A section $f$ over $V$ may be pulled back to sections over the varieties $V^{\prime}$. Since every section over every $V^{\prime}$ is of finite order, we can obtain a dense set of points of $V$ which map via $f$ to points of finite order 
in the fibres over $V$. To show $f$ is torsion, we must show these orders are bounded. We do this by proving a theorem giving a uniform bound for orders of torsion points on fibres with complex multiplication (Theorem 2 below). The finiteness of the Mordell-Weil group of the generic fibre then follows.

For $u$ in $V$, write $Q_{u}=\left(A_{u}, C_{u}, \theta_{u}, t_{1}(u), \ldots, t_{s}(u)\right)$ for the fibre over $u$. The fibre system $\mathcal{F}$ is defined over a number field $k_{\Omega}$ of finite degree such that for every $u \in V, k_{\Omega}(u)$ is the field of moduli of $Q_{u}$ (see [5]). Call $Q_{u}$ a " $C M$ fibre" if $A_{u}$ is isogenous to $A_{1} \times \cdots \times A_{t}$, where $A_{i}$ has complex multiplication by a $C M$-field of degree $2 \cdot \operatorname{dim}\left(A_{i}\right)$, for $i=1, \ldots, t$ (thus, $A_{u}$ has $C M$ in the sense of $[6])$.

THEOREM 2. Let $k$ be any subfield of $\mathbb{C}$ which is finitely generated over $\mathrm{Q}$ and contains $k_{\Omega}$. There is a constant $B$, depending only on the field $k$ and the fibre system $\mathcal{F}$, and independent of the choice of $C M$-fibre $Q_{u}$, so that $\left|A_{u}(k(u))_{\text {torsion }}\right| \leq B$.

The proof of Theorem 2 requires Shimura's Main Theorem of Complex Multiplication.

\section{REFERENCES}

1. J.-I. Igusa, On the structure of a certain class of Kaehler varieties, Amer. J. Math. 76 (1954), 669-678.

2. Y. Matsushima and G. Shimura, On the cohomology groups attached to certain vector valued differential forms on the product of the upper half planes, Ann. of Math. (2) 78 (1963), 417-449.

3. G. Shimura, On analytic families of polarized abelian varieties and automorphic functions, Ann. of Math. (2) 78 (1963), 149-192.

4. 81 (1965), 124-165.

5. ___ Moduli and fibre systems of abelian varieties, Ann. of Math. (2) 83 (1966), 294-338.

6. - On canonical models of arithmetic quotients of bounded symmetric domains, Ann. of Math. (2) 91 (1970), 144-222.

7. Introduction to the arithmetic theory of automorphic functions, Iwanami Shoten, Tokyo and Princeton Univ. Press, Princeton, 1971.

8. T. Shioda, On elliptic modular surfaces, J. Math. Soc. Japan 24 (1972), 20-59.

9. A. Silverberg, Ph.D. thesis, Princeton Univ., 1984.

Department of Mathematics, Princeton University, Princeton, New JERSEY 08544 43210

Current address: Department of Mathematics, Ohio State University, Columbus, Ohio 